
\title{
$\begin{array}{ll}\text { Research Square } & \text { Preprints are preliminary reports that have not undergone peer review. } \\ \text { They should not be considered conclusive, used to inform clinical practice, } & \text { or referenced by the media as validated information. }\end{array}$
}

\section{Features of Sepsis With Worse Outcomes in the ICU Admissions: a Cohort Study From Quick Diagnostic Tests}

Dao-Ming. Tong ( $\square$ tongdaoming@163.com )

Affiliated Shuyang Hospital of Xuzhou Medical University

Ye-Ting. Zhou

Affiliated Shuyang Hospital of Xuzhou Medical University

Shao-Dan. Wang

Affiliated Shuyang Hospital of Xuzhou Medical University

Guang-Sheng. Wang

Affiliated Shuyang Hospital of Xuzhou Medical University

Yuan-Wei. Wang

Affiliated Shuyang Hospital of Xuzhou Medical University

\section{Ying. Wang}

Affiliated Shuyang Hospital of Xuzhou Medical University

Jin-Jin. Zhou

Affiliated Shuyang Hospital of Xuzhou Medical University

\section{Research Article}

Keywords: Sepsis, Critically ill, Community-acquired pneumonia, Systemic inflammatory response syndrome, Organ failure, Mortality

Posted Date: October 21st, 2021

DOI: https://doi.org/10.21203/rs.3.rs-936853/v1

License: (a) (i) This work is licensed under a Creative Commons Attribution 4.0 International License. Read Full License 


\section{Abstract}

Background: Sepsis is still a global public health priority. We aimed to investigate the features of sepsis with worse outcomes in a single ICU of China..

Methods: We enrolled patients with acute critically ill with sepsis from ICU in China (Jan 1, 2018 to Nov $30,2019)$. All patients underwent quick diagnostic tests before and after on admission. We collected data on the features of sepsis cases from hospital medical records and follow-up information. Outcomes were compared between patients who were survivors and those who were not.

Results: Of 520 ICU admissions, $214(41.2 \%)$ critically ill adults with sepsis were analyzed. The median age was 66 years (range 18-91 years) and 140 (65.4\%) patients were males. 144 (78.3\%) communityacquired pneumonia in 184 pneumonia cases were identified by quick chest CT and need mechanically ventilation in ICU. The rate of community- acquired sepsis was $73.8 \%$ (158/214) and was associated with a high risk of dynamic profile of inflammatory mediators for nonsurvivors. The initial common symptoms of sepsis were fever (42.5\%), altered mental status/delirium (41.1\%), acute coma (34.1\%), and cough/dyspnea (29.9\%). Based on data with significant univariate at initial 24 hours in ICU, the multivariate logistic analysis revealed a higher SIRS score (OR,4.7; $95 \% \mathrm{Cl}, 2.004-14.37)$, a higher SOFA score which mainly showed higher prevalent lungs and brain failure (OR, 1.7; $95 \% \mathrm{Cl}, 1.299-2.298)$, and a lower GCS score (OR, $0.8 ; 95 \% \mathrm{Cl}, 0.673-0.987)$ were established as early risk factors for sepsis in nonsurvivors. The mortality of sepsis was $55.6 \%$ at 28 days.

Conclusions and relevance: Sepsis cases are mainly from community-acquired pneumonia. Severe SIRS and lungs/brain failure are related to the high mortality of sepsis.

\section{Introduction}

Sepsis is a life-threatening organ dysfunction that is caused by a dysregulated host response to infection [1]. However, the sepsis-met sepsis-3 criteria, also the most frequent complication of critically ill adults in the ICU, has become the leading cause of morbidity and mortality worldwide [2,3]. Moreover, the WHO estimates 30 million new cases of sepsis and approximately 6 million deaths per year in high-income countries worldwide, and recognizes sepsis as a global public health priority [4]. Unfortunately, this data may be severely underestimated because its statistics did not have the data from China and in lowincome countries worldwide. Furthermore, this sepsis has been largely ignored by clinical workers and the public because its onset is often hidden behind various critical illnesses and is unlike coronavirus disease 2019 (COVID-2019) patients with sepsis with outbreak transmission [5, 6]. In the present study, we aimed to determine the features of worse outcomes of critically ill patients with sepsis in a single ICU of China, including epidemiological, clinical complications, radiological findings, laboratory data, and treatment. Moreover, our cohort study is from quick diagnostic tests (quick systemic CT scans and quick clinical tests). We hope that our study results will provide new evidence for the update of sepsis and achieve effective treatment. 


\section{Methods}

\section{Study setting and population}

This study included critically ill adults who were admitted to a general ICU at the Affiliated Shuyang Hospital of Xuzhou Medical University in China. The current study recruited critically ill adults who were admitted between Jan 1, 2018, and Nov 30, 2019 (before COVID-19 outbreak), with a length of stay of more than 24 hours. We examined the electronic medical records of patients who were verified as having critically ill events with available records. We also excluded possible critically ill patients who did not have data in their medical records due to either death or transport out of the ICU within the first 24 hours. The study was approved by the ethical committee on clinical research of the Affiliated Shuyang Hospital of Xuzhou Medical University. Because the study involved only a review of records obtained as a part of routine medical care, it did not require written information consent from all patients.

\section{Patient identification}

All patients underwent quick systemic CT scans (brain, thorax, and abdominal) before on admission. On quick chest CT tests, we identified community- acquired pneumonia (the typical pneumonia of chest CT images including bilateral multiple lobular and subsegmental areas of consolidation). The communityacquired sepsis was diagnosed by an acute change in the total Sequential Organ Function Assessment (SOFA) scores $\geq 2$ points consequent to the community-acquiretherd pneumonia and/or systemic inflammatory response syndrome (SIRS).

A SIRS mainly involves massive inflammatory cytokine release [7]. More recently, this response has been called a cytokine storm or cytokine release syndrome, which is a life-threatening systemic inflammatory syndrome [8]. Furthermore, SIRS has been recognized as a clinical manifestation of the host response to suspected infection. Thus, we used SIRS criteria $\geq 2$ to identify infection events within first 0.5-1 hour in ICU. The clinical manifestations of SIRS include the following 4 points: (1) temperature $>38^{\circ} \mathrm{C}$ or $<36^{\circ} \mathrm{C}$; (2) heart rate $>90$ beats per minute;(3) tachypnea greater than 20 respirations per minute or Pco2 less than $32 \mathrm{mmHg}$; (4) white blood cell count $>12.0 \times 10^{9} / \mathrm{L}$ or $<4.0 \times 10^{9} / \mathrm{L}$, or $>10 \%$ band forms. The identified criteria for SIRS must be at least $\geq 2$ points/scores ( $\geq 3$ points/scores= severe). In addition, this inflammatory syndrome also involves other inflammatory mediators in the blood (e.g., interleukin-6, Ddimer and C-reactive protein). Therefore, we also quick tested these inflammatory mediators.

Sepsis must present one or more acute organ dysfunctions caused by infection. Acute organ dysfunction is defined as equivalent to a sequential [sepsis-related] organ function assessment (SOFA) score $\geq 2$ for a particular organ [with $>4$ scores indicating a multiple organ dysfunction syndrome (MODS)]. For example, a SOFA score $\geq 2$ for acute respiratory failure/ARDS indicates $\mathrm{PaO} 2 /$ Fio $2<300 \mathrm{mmHg}$ or Sp02<93\% on minimal $2 \mathrm{~L} / \mathrm{min}$ supplemental oxygen. The SOFA criteria for other organ failures are reported in document [9].

\section{Data collected}


The collected clinical profile for this study performed in the ICU included patient demographics, comorbidities, time from critical illness to ICU admission, presenting symptoms, vital sign data, acute organ dysfunction, laboratory tests, cranial and thorax/ abdominal CT scans findings, and treatment during the ICU stay. Laboratory tests included complete blood count, coagulation data (D-dimer and fibrinogen), liver and renal function, cytokines and other inflammatory mediators, SIRS score, SOFA score, mechanical ventilation, length of stay (LOS) in the ICU, and outcomes.

The outcome events were reviewed by two of the investigators (the first and fourth authors). To investigate the outcomes of patients during the first 72 hours and at 28 days, the survival results were determined from the hospital records and followed up to 28 days.

Based on the inclusion criteria for sepsis after critically ill events, the study data were divided into the sepsis survivor group and nonsurvivor group for statistical analysis.

\section{Statistical methods}

The results for each group are expressed as the mean \pm standard deviation (SD) or median (IQR), and $n$ (\%) indicates qualitative values. Fisher's exact test and the Mann-Whitney $U$ test were used to examine the relationship between baseline patient variables. Continuous variables were compared using Student's $t$ test. Multivariate-adjusted odds ratios (ORs) and 95\% confidence intervals (Cls) were estimated using a logistic regression model if they were significant according to the univariate analysis. Differences between patients were considered significant if the $p$-value was $<0.05$. Statistical calculations were performed using a proprietary, computerized statistics package (SPSS 17.0.).

\section{Results}

A total of 520 acute critically ill adult patients were recruited. After ruling out patients who did not meet the eligibility criteria for the study design, infection was confirmed by a brain-chest-abdomen CT scan before on admission. Finally, $214(41.2 \%)$ critically ill patients with sepsis at ICU admission were identified by the sepsis-3 criteria (Figure 1).

The clinical characteristics of the 214 sepsis patients are shown in Table 1. The median age was 66.7 years (range 18-91 years) and 140 (65.4\%) patients were males. Of these patients, the most common comorbidity was hypertension (45.8\%), followed by previous cardiac-cerebral vascular disease (33.6\%), current acute traumatic brain injury events (27.1\%), and current intracranial hemorrhage events $(18.7 \%)$. The most common initial presenting symptoms were fever [91 (42.5\%)], altered mental status/delirium [88 (41.1\%)], acute coma [73 (34.1\%)], and cough/dyspnea [64 (29.9\%)]. The median time from onset to ICU admission was 3.2 hours (range, $0.5-240 \mathrm{~h}$ ). 
Table 1

Clinical characteristics in patients with sepsis $(n=214)$

\begin{tabular}{|c|c|c|c|c|}
\hline & $\begin{array}{l}\text { Total } \\
(n=214)\end{array}$ & $\begin{array}{l}\text { Non- } \\
\text { survivors } \\
(n=119)\end{array}$ & $\begin{array}{l}\text { Survivors } \\
(\mathrm{n}=95)\end{array}$ & $\begin{array}{l}p \\
\text { Value }\end{array}$ \\
\hline Age (years, mean $\pm S D$ ) & $66.7 \pm 16.1$ & $67.7 \pm 15.7$ & $65.4 \pm 16.5$ & 0.297 \\
\hline Male gender $(n, \%)$ & $140 \rrbracket 65.4 \rrbracket$ & $79 \rrbracket 66.4 \rrbracket$ & $61 \rrbracket 64.2 \rrbracket$ & 0.774 \\
\hline \multicolumn{5}{|l|}{ Comorbidities } \\
\hline Hypertension( $n, \%)$ & $98(45.8)$ & $54(45.4)$ & $44(46.3)$ & 1.000 \\
\hline $\begin{array}{l}\text { Previous cardiac-cerebral vescular } \\
\text { disease },(n, \%)\end{array}$ & 72(33.6) & $39(32.8)$ & $33(34.7)$ & 0.773 \\
\hline \multicolumn{4}{|l|}{ Diabetes( $n, \%)$} & 0.360 \\
\hline 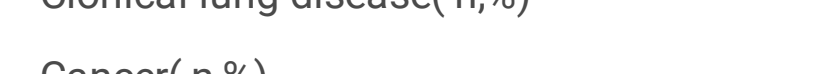 & $30(14.0)$ & $11(9.0)$ & $19(20.0)$ & 0.029 \\
\hline (2) & $19(0.9)$ & $12(10.1)$ & $7(7.4)$ & 0.630 \\
\hline (1) & $58(27.1)$ & $34(28.6)$ & $24(25.3)$ & 0.644 \\
\hline Initial presenting symptoms & $40(18.7)$ & $31(26.1)$ & $9(9.5)$ & 0.002 \\
\hline \multicolumn{5}{|l|}{ Fever,( $n, \%)$} \\
\hline Alterd mental status/deliriun, $(n, \%)$ & $80(111)$ & $57(170)$ & $21(226)$ & 00 \\
\hline Acute coma, $(n, \%)$ & $00(41.1)$ & (4) & $01(0<.0)$ & $0.0<0$ \\
\hline Cough/dyspnea,(n,\%) & 73(34.1) & 49(41.2) & 24(25.3) & 0.020 \\
\hline Chest/abdominal pain, $(n, \%)$ & 64(29.9) & $44(37.0)$ & $20(21.1)$ & 0.016 \\
\hline Dizziness/headache,(n,\%) & $12(5.6)$ & $6(5.0)$ & $6(6.3)$ & 0.769 \\
\hline Other (n\%) & $11(5.1)$ & $5(4.2)$ & $6(6.3)$ & 0.554 \\
\hline Acute nnoumonia/lung infocti & $5(2.3)$ & $3(2.5)$ & $2(2.1)$ & 1.000 \\
\hline Community-accuired cencis & 184(86.0) & $99(83.2)$ & $85(89.5)$ & 0.236 \\
\hline 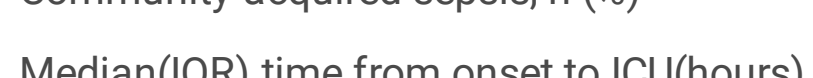 & $158(73.8)$ & $88(73.9)$ & $70(73.7)$ & 1.000 \\
\hline Median $(10 \mathrm{~B}) \mathrm{gcc}$ cenro & $3.2(0.5-240)$ & $2.7(0.5-$ & $5.5(0.5-240)$ & 0.012 \\
\hline 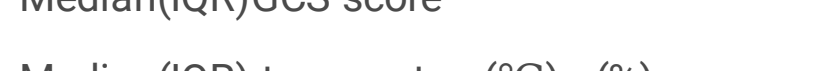 & $13(5-15)$ & & $13(5-15)$ & 0.044 \\
\hline Median(IQR) temperature $\left({ }^{\circ} \mathrm{C}\right) \mathrm{n}(\%)$ & 37.5(35.3- & $12(5-15)$ & $36.9(35.3-$ & 0.075 \\
\hline Median(IQR)arterial pressure(mmHg) & & $\begin{array}{l}37.8(36- \\
40.0)\end{array}$ & & 0.266 \\
\hline Median(IQR)Heart rate(beats/min) & $93(40-160)$ & $93(40-160)$ & $98.7(90-130)$ & 0.354 \\
\hline
\end{tabular}




\begin{tabular}{|lllll|} 
Median(IQR)Respiratory rate(breaths/mim) & $102(58-107)$ & $102(70-$ & $102(58-148)$ & 0.818 \\
MODS, n (\%) & $27(0-45)$ & $107)$ & $27(0-45)$ & 0.007 \\
LOS (days,IQR) & $206(96.3)$ & $27(5-35)$ & $87(991.6)$ & 0.001 \\
Mortality at 28 days,n (\%) & $8.8(1-127)$ & $119(100.0)$ & $9.5(2-127)$ & 0.300 \\
& $119(55.6)$ & $7.5(1-90)$ & NA & \\
& & NA & & \\
\hline
\end{tabular}

Abbreviations: ICU, intensive care unit; GCS, Glasgow Coma Scale; SOFA, Sequential [sepsis-related] Organ Function Assessment; LOS, length of stay; IQR;interquartile range $\mathbb{M} \mathrm{MDS}$, multiple organ dysfuunction syndrome.

Among the 214 critically ill patients with sepsis, 184 (86.0\%) pneumonia patients, including $78.3 \%$ (144/184) community-acquired pneumonia (Figure 2) and 21.7\% nosocomial- acquired pneumonia, were diagnosed by chest CT and/or digital radiography (DR) and need mechanically ventilation in ICU. Of these, 158 (73.8\%) community-acquired sepsis patients were diagnosed based on SOFA scores $\geq 2$ points. In all sepsis patients, the risk of death was $16.8 \%(36 / 214)$ at the initial 72 hour point and $55.6 \%$ $(119 / 214)$ at 28 days.

\section{Dynamic profile of laboratory findings in patients with sepsis}

The data of the surviving or nonsurviving sepsis patients are described in Table 2. The laboratory findings in the survivors were from the worst value during the clinical course in the ICU, but the major critical values in the nonsurvivors were tracked before death. The main dynamic findings during sepsis progression included 6 laboratory parameters, which were from within the initial 1 days to 24 days after ICU admission at 3-day intervals. The interleukin-6 (IL-6), D-dimer, C-reactive protein (CRP), and SpO2 levels and the SIRS and SOFA scores were higher in the nonsurvivors than in the survivors (Figure 3 ). In addition, we found that nonsurviving sepsis patients were more likely to present with higher qSOFS scores, elevated procalcitonin, elevated creatinine, elevated blood glucose, and elevated lactic acid than survivors (all $p<0.05$ ). However, the white blood cell counts and neutrophil counts were similarly high between the nonsurvivors and survivors. 
Table 2

Laboratory findings in patients with sepsis who died and survivor $(n=214)$

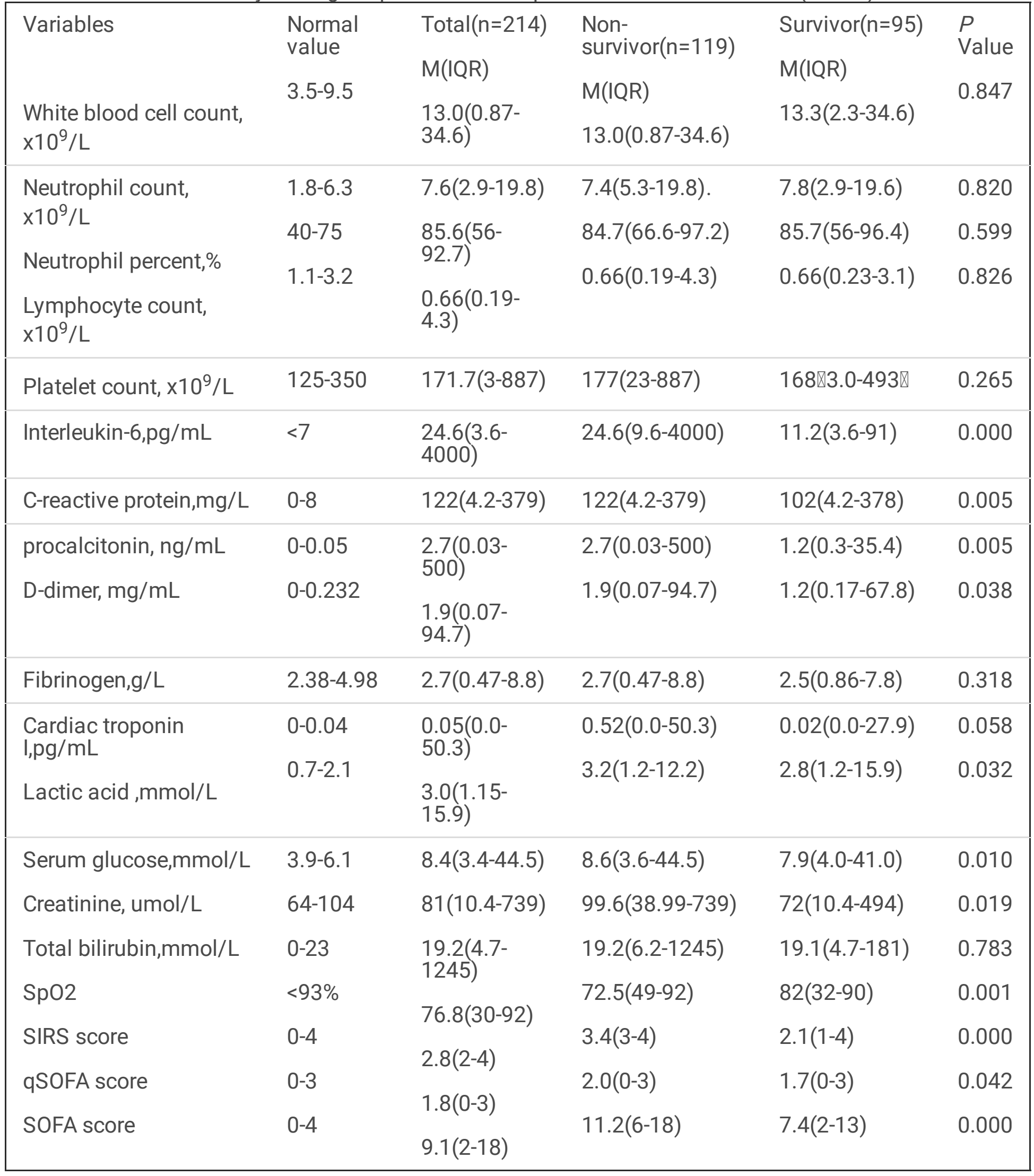

Abbreviations: SPO2, saturation of arterial oxygen; SISS, systemic inflammatory response syndrome; SOFA, Sequential [sepsis-related] Organ Function Assessment; qSOFA, quick SOFA. 
However, by logistic regression analysis, only a severe SIRS (OR, 4.7; 95\% Cl, 2.004-14.37), a higher SOFA score which mainly indicate lungs and brain failure (OR, 1.7; $95 \% \mathrm{Cl}, 1.299-2.298)$, and a lower GCS score (OR, 0.8; 95\% Cl, 0.673-0.987), were established as early risk factors for sepsis in nonsurvivors. (Table 3 )

Table 3

Logistic regression analysis to identify the death risk factors of sepsis patients in ICU $(n=214)$

\begin{tabular}{|c|c|c|c|c|}
\hline Variables & $\begin{array}{l}\text { Non-survivors } \\
(\mathrm{n}=119)\end{array}$ & $\begin{array}{l}\text { Survivors } \\
(n=95)\end{array}$ & $\begin{array}{l}\text { OR } \\
(95 \% \mathrm{Cl})\end{array}$ & $\begin{array}{l}P \\
\text { Value }\end{array}$ \\
\hline SIRS score (mean $\pm S D)$ & $5.3 \pm 0.65$ & $3.8 \pm 0.68$ & $4.7(2.004-14.37)$ & 0.000 \\
\hline SOFA score (mean $\pm S D)$ & $11.3 \pm 2.9$ & $7.3 \pm 2.4$ & $1.7(1.299-2.298)$ & 0.000 \\
\hline GCS score (mean $\pm S D)$ & $10.4 \pm 3.3$ & $11 . .7 \pm 3.8$ & $0.8(0.673-0.987)$ & 0.035 \\
\hline
\end{tabular}

Abbreviations: SIRS, systemic inflammatory response syndrome; SOFA, Sequential [sepsis-related] Organ Function Assessment; ICU, intensive care unit.

Acute respiratory failure/ARDS $(93.5 \%, 200 / 214)$ and acute brain failure $(94.4 \%, 202 / 214)$ were the most frequent life-threatening organ dysfunctions for sepsis, followed by septic shock (47.7\%, 102/214), and acute kidney failure $(33.2 \%, 71 / 214)$. Moreover, MODS was associated with up to $96.3 \%(206 / 214)$ and $57.0 \%$ mortality (Figure 4 ).

\section{Discussion}

The prevalence of sepsis in the last 30 years has been reported to range from $21.7-51.5 \%$ of all patients admitted to the ICU $[10,11]$, about 30-42.8\% community-acquired pneumonia associated sepsis $[2,3,11$, $12]$, with a mortality rate of $23-48.2 \%$ in the ICU $[2,3,11], 81.7 \%$ of septic patients with delirium in the ICU develop acute respiratory distress syndrome (ARDS), requiring intubation and mechanical ventilation [13]. Although sepsis has been frequently studied, its clinical features and risk factors of patients who were non-survivors still need clarification. Undoubtedly, early diagnosis and treatment can reduce the mortality of sepsis. In the present study, so we used quick diagnosis tests, including early systemic CT scans and quick clinical tests to screen infection and sepsis. We found that the features of sepsis were mainly from community-acquired sepsis (73.8\%), and most of them with community-pneumonia (78.3\%). These data are higher than previous study [14], and the features are similar to the current outbreak of COVID-19 sepsis $[9,15]$.

More importantly, we found that acute respiratory failure/ARDS (93.5\%) and acute brain failure (94.4\%) were the most common life-threatening organ dysfunctions in most of the sepsis cases, with a $55.6 \%$ risk of death within the initial 28 days in the ICU. 
It should be noted, however, that critically ill ICU patients with sepsis with a high risk of death in our study were more likely to present with a higher SIRS score, a higher SOFA score, a higher qSOFA score, elevated interleukin-6, elevated D-dimer, lower GCS score and lower Sp02, elevated CRP and procalcitonin, elevated creatinine, elevated blood glucose, and elevated lactic acid. Notably, by multivariate regression analysis, only higher SIRS scores and higher SOFA scores (MODS) were related to high mortality in critically ill patients with sepsis.

Several possible interpretations of the results for sepsis with high mortality are as follows. First, MODS due to sepsis resulting in high mortality has been recognized [4, 14, 16-19], and previous studies have shown that sepsis patients with MODS are more likely to exhibit ARDS $[4,17-19]$ and acute brain dysfunction [13, 20-22], which is related to their vulnerability to hypoxia/SIRS [7, 13, 19-22]. Our data showed that current sepsis had MODS and that over $90.0 \%$ of patients with sepsis had acute respiratory failure/ARDS. The features of ARDS are mainly acute pneumonia/pulmonary infection due to a dysregulated immune response leading to higher inflammation in the lungs, followed by the development of pulmonary edema, hyaline membrane formation, and respiratory failure [19, 23]. Moreover, acute respiratory failure/ARDS plays a pivotal role in the development of MODS in septic events $[7,13,14,18]$; epithelial damage and SIRS are the main pathophysiological features of ARDS and play an important role in distinguishing the hyperinflammatory subtypes of ARDS [19] .

Another possibility is that acute brain failure is also ranked as the leading organ dysfunction and for the host response to hyperinflammation, which is more likely to be due to hypoxic/SIRS [13, 14, 16, 20-22], especially with a cause of acute pneumonia/pulmonary infection $[14,17,18]$. Similar to the current outbreak of COVID-19 pneumonia, the mechanisms of bacterial pneumonia are also related to angiotensin conversion enzyme 2 (ACE2)- involved inflammatory response/cascade [24, 25]. Furthermore, blood- brain barrier (BBB) leakage is also due to an overwhelming release of proinflammatory cytokines and anti- inflammatory factors [26-28]. SIRS is responsible for extensive BBB leakage and even brain edema and cerebral ischemic injury as well as cell death [26-29]. Our current study confirmed that SIRS is a very high risk factor for dying from sepsis, supporting that it is a life-threatening systemic inflammatory syndrome $[8,30]$.

Strengths in this study, we are a first to report that a brain-chest-abdomen CT scan before arriving at the hospital to identify early infection site which is a visible really infection. Severe SIRS and severe lungs/brain failure could use to predict the risk of sepsis with worse outcomes,

However, some limitations of our design must be addressed. One limitation is the single central design, which may present a selection bias. However, this hospital is responsible for the medical care of the prefecture's population of approximately 1.8 million people, including an urban center and 38 villages and towns. This hospital ICU is a unique regional ICU (open 21-bed) with an emergency center (a total of 10 ambulances) that is responsible for all critically ill referrals in this prefecture. Thus, we believe that this sample does not lead to a large bias. The second limitation of this study is that organ dysfunction meeting the SOFA criteria was evaluated only according to old criteria. Although we made some changes, 
the observed SOFA scores may still be underestimated. In addition, although the prognosis for sepsis was related to SIRS and MODS, further prospective multicenter studies are needed for some of the intermediary links.

\section{Conclusion}

The vast majority of sepsis cases are from highly prevalent community-acquired pneumonia and with high risk of death. Severe SIRS and high prevalent lungs/brain failure are related to the high mortality of sepsis, suggesting that rapid antibiotic IV within the initial 1.0 hours in the ICU may improve patients' outcomes.

\section{Abbreviations}

ICU: Intensive care unit; GCS, Glasgow Coma Scale; LOS, length of stay; IQR; interquartile range $\mathbb{M O D S}$, multiple organ dysfuunction syndrome. SPO2, saturation of arterial oxygen; SISS, systemic inflammatory response syndrome; SOFA, Sequential [sepsis-related] Organ Function Assessment; qSOFA, quick SOFA.

\section{Declarations}

\section{Contributors}

Dr. Tong had full access to all of the data in the study and takes responsibility for the integrity of the data and the accuracy of the data analysis.

Study concept and design:Tong, Zhou, Acquisition, analysis, or interpretation of data:Tong, Zhou

Drafting of the manuscript:Tong.

Statistical analysis:Tong.

Critical revision of the manuscript for important intellectual content:All authors..

\section{Funding}

This work is supported by a grant from the Medical Research Council and the Affiliated Shuyang Hospital of Xuzhou Medical University (from the National Clinical Key Specialty Construction Project (2020-0019-N -0003), China.

\section{Declaration of Interests}

No potential conflicts of interest were reported For all .authors 
Availability of data and materials

The datasets used and/or analyzed during the current study are available from the corresponding author on reasonable request.

\section{Ethics approval and consent to participate}

Approval was provided by the Institutional Review Board of the Shuyang People's Hospital, China.

\section{Consent for publication}

Because the study involved only a review of records obtained as a part of routine medical care, it did not require written information consent from all patients for publication of this article and any accompanying images. Informed consent was waived by ethics committee of Shuyang Hospital.

\section{Acknowledgments}

We would like to thank everyone who contributed to the study, namely, the nurses and physicians who recorded the patient data and the related senior experts for reviewing the medical records in the ICU.

\section{References}

1. SingerM, DuetschmenCS, SeymourCW, et al. TheThird International Consensus Definitions for Sepsis and Septic Shock (Sepsis-3). JAMA. 2016;315:801-810.

2. Park DW, Chun BC, Kim JM, et al. Epidemiological and Clinical Characteristics of CommunityAcquired Severe Sepsis and Septic Shock: A Prospective Observational Study in 12 University Hospitals in Korea. J Korean Med Sci.2012; 27:1308- 1314.

3. Angus DC, Linde-Zwirble WT, Lidicker J, et al. Epidemiology of severe sepsis in the United States: analysis of incidence, outcome, and associated cost of care. Crit Care Med. 2001,29 (7):1303 -1310.

4. Konrad Reinhart, Daniels, Niranjan Kissoon, et al. Recognizing sepsis as a global health priority-A WHO resolution.N Engl J Med 2017;377:414-417.

5. Morens DM, Daszak P, Taubenberger JK. Escaping Pandora's box-another novel coronavirus. N Engl J Med. 2020; 382: 1293-1295.

6. Wu Z, McGoogan JM. Characteristics of and important lessons from the coronavirus disease 2019 (COVID-19) outbreak in China: summary of a report of 72314 cases from the Chinese Center for Disease Control and Prevention. JAMA. 2020; 323:1239- 1242.

7. Bone RC. Toward a theory regarding the pathogenesis of the systemic inflammatory response syndrome: what we do and do not know about cytokine regulation. Crit Care Med. 1996; 24: 163-172.

8. David C. F, Carl H. J. Cytokine Storm. N Engl J Med 2020; 383:2255-2273.

9. Vincent JL, Moreno R, Takala J, et al. The SOFA (Sepsis-related Organ Failure Assessment) score to describe organ dysfunction/ failure. On behalf of the Working Group on Sepsis-Related Problems of 
the European Society of Intensive Care Medicine. Intensive care medicine.1996;22(7):707-10.

10. Padkin A, Goldfrad C, Brady AR, Young D, Black N, Rowan K. Epidemiology of severe sepsis occurring in the first 24 hrs in intensive care units in England, Wales, and Northern Ireland. Crit Care Med. 2003;31:2332-2338.

11. Blanco J, Muriel-Bombín A,Sagredo V, et al. Incidence, organ dysfunction and mortality in severe sepsis: a Spanish multicentre study. Crit Care. 2008;12:R158. doi: 10.1186/cc7157

12. Westphal GA, Pereira AB, Fachin SM,et al. Characteristics and outcomes of patients with communityacquired and hospital- acquired sepsis. Rev Bras Ter Intensiva. 2019;31(1):71-78.

13. Ely EW, Shintani A, Truman B, et.al. Delirium as a predictor of mortality in mechanically ventilated patients in the intensive care unit.JAMA.2004;291:1753-1762

14. Beutz MA, Abraham E. Community-acquired pneumonia and sepsis. Clin Chest Med. 2005;26(1):1928.

15. Salehi S, Abedi A, Balakrishnan S et al Coronavirus Disease 2019 (COVID-19): A Systematic Review of Imaging Findings in 919 Patients. AJR Am J Roentgenol,2020;215:87-93

16. Esen F, Orhun G, Özcan PE, et al. Diagnosing acute brain dysfunction due to sepsis. Neurological Sciences .2020; 41:25-33

17. Iskander KN, Osuchowski MF, Stearns-Kurosawa DJ, et al. Sepsis: Mutiple abnormalities, heterogeneous responses, and evolving understanding. Physiol Rev 2013, 93:1247-1288.

18. Bral AL, Cerra FB. Multiple organ failure syndrome in 1990, systemic inflammatory response and organ dysfunction. JAMA. 1994,27:226-233.

19. Spadaro S., Park M., Turrini C. Biomarkers for Acute Respiratory Distress syndrome and prospects for personalised medicine. J Inflamm. 2019;16:1

20. Sonneville R,de Montmollin E, Poujade J, et al. Potentially modifiable factors contributing to sepsisassociated encephalo -pathy. Intensive Care Med. 2017;43:1075-1084.

21. Gofton TE, Young GB.Sepsis-associated encephalopathy.Nat Rev Neurol.2012, 8:55766.10.1038/nrneurol.2012.183

22. Shulyatnikova T, Verkhratsky A. Astroglia in sepsis associated encephalopathy. Neurochem Res.2019, 18.

23. Fan E, Brodie D, Slutsky AS. Acute Respiratory Distress Syndrome: Advances in Diagnosis and Treatment. JAMA. 2018 20; 319(7): 698-710

24. Gaddam RR, Chambers S, Bhatia M. ACE and ACE2 in Inflammation: A Tale of Two Enzymes. Inflamm Allergy Drug Targets. 2014;13(4):224-34.

25. Sodhi CP, Nguyen J, Yamaguchi Y, et al. A Dynamic Variation of Pulmonary ACE2 Is Required to Modulate Neutrophilic Inflammation in Response to Pseudomonas aeruginosa Lung Infection in Mice.J Immunol. 2019 1;203(11):3000-3012.

26. Ilaria Alice Crippa,Carles Subirà,Jean-Louis Vincent, et al. Impaired cerebral autoregulation is associated with brain dysfunction in patients with sepsis. Crit Care. 2018; 22: 327. 
27. Shulyatnikova T, Verkhratsky A. Astroglia in sepsis associated encephalopathy. Neurochem Res.2019, 18.

28. Kaml GJ, Davis KA. Surgical Critical Care for the Patient with Sepsis and Multiple Organ Dysfunction. Anesthesiol Clin. 2016;34:681-696.

29. Sharshar T, Carlier R, Bernard F, et al., Brain lesions in septic shock: a magnetic resonance imaging study, Intensive Care Medicine,2007; 33:798-806

30. Kang S, Tanaka T, Inoue H, et al. IL-6 trans-signaling induces plasminogen activator inhibitor-1 from vascular endothelial cells in cytokine release syndrome. Proc Natl Acad Sci U S A. 2020 8;117:22351 -22356 .

\section{Figures}




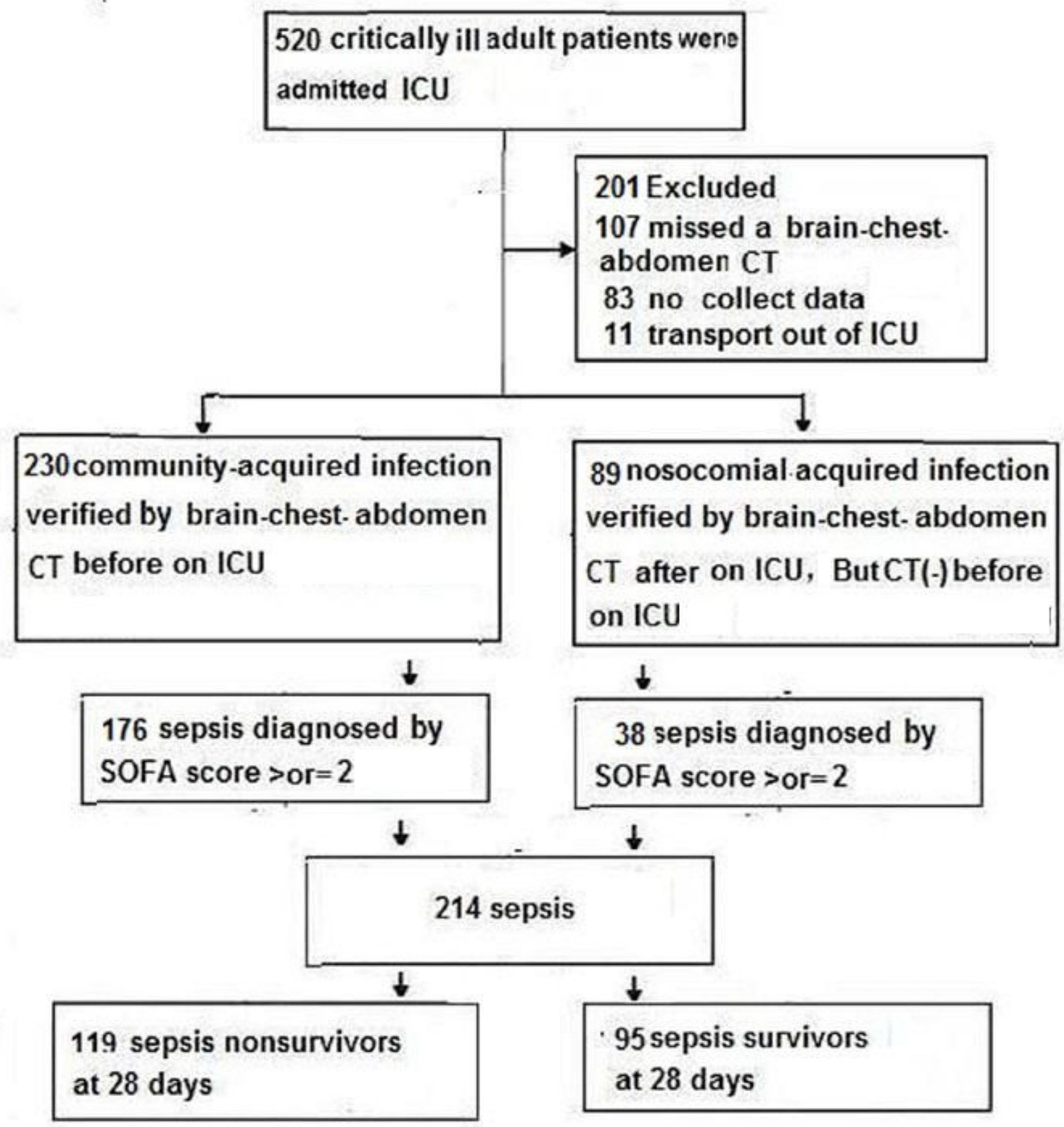

Figure 1

Study profile. Acute critically ill adult patients who were undergone a brain- chest-abdomen computed tomograph(CT) scans before arriving at the ICU were selected as an eligibility criteria. 


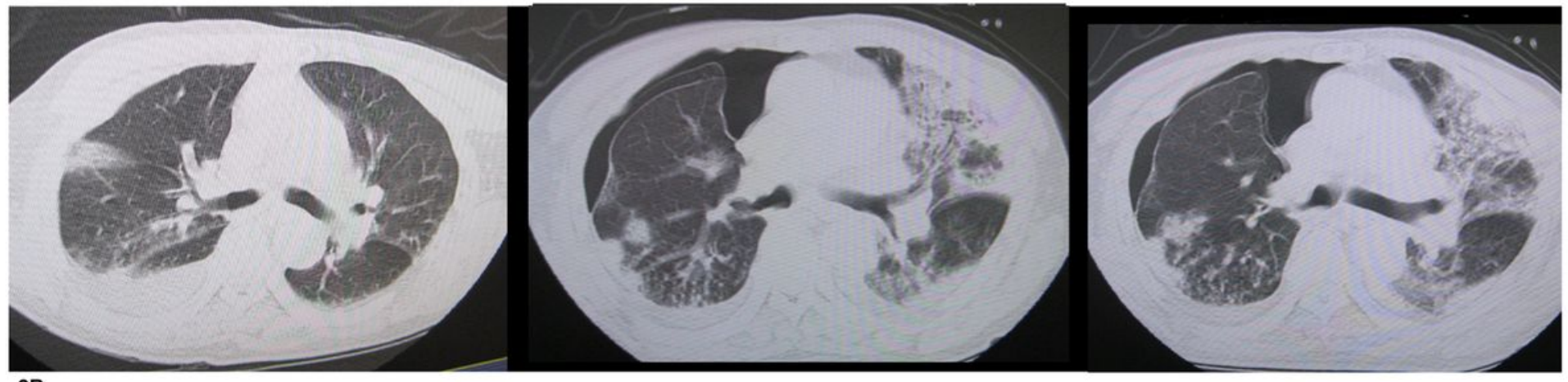

2B

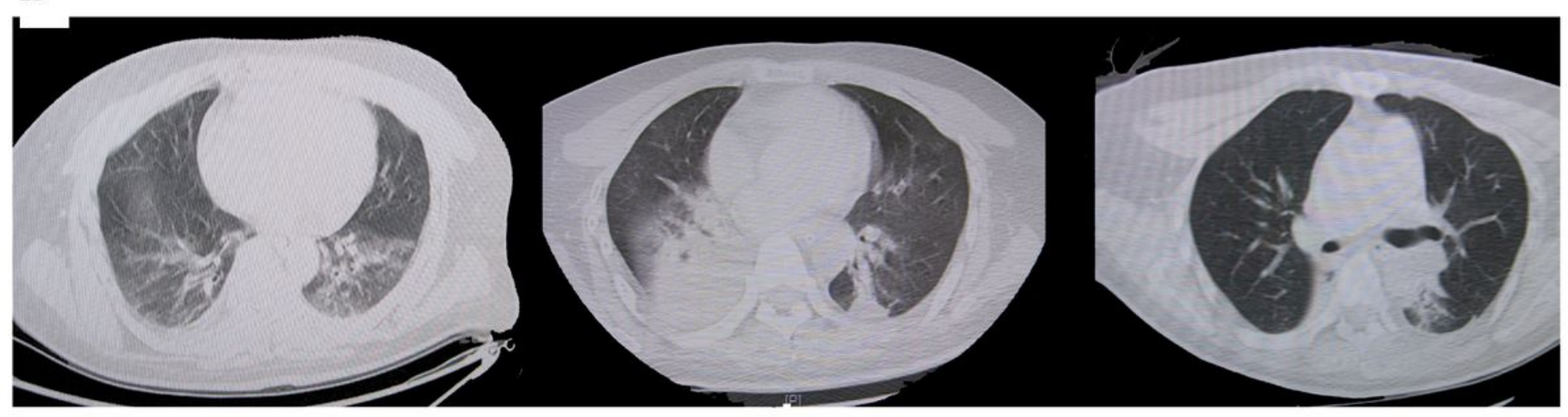

Figure 2

The features of the chest computed tomographic (CT) images. Figure $2 \mathrm{~A}$ is from a patient with bacterial sepsis and ARDS. On days 2, 7, and 17 after symptom onset, chest CT showed progressive bilateral pneumonia deterioration with a large area of ground glass opacities in the left lobe, and due to resistance to antibiotics, the patient died. Figure $2 \mathrm{~B}$ is from a patient with sudden delirium who was admitted to the ICU. On day 2 and day 7 after symptom onset, chest CT showed bilateral pneumonia in the lungs. On day 3 , the patient was in coma and respiratory failure requiring respiratory intubation, and bacterial sepsis was diagnosed. On day 10 , chest CT showed that the bilateral lesions had improved by using antibiotics for treatment, and the patient finally recovered. 

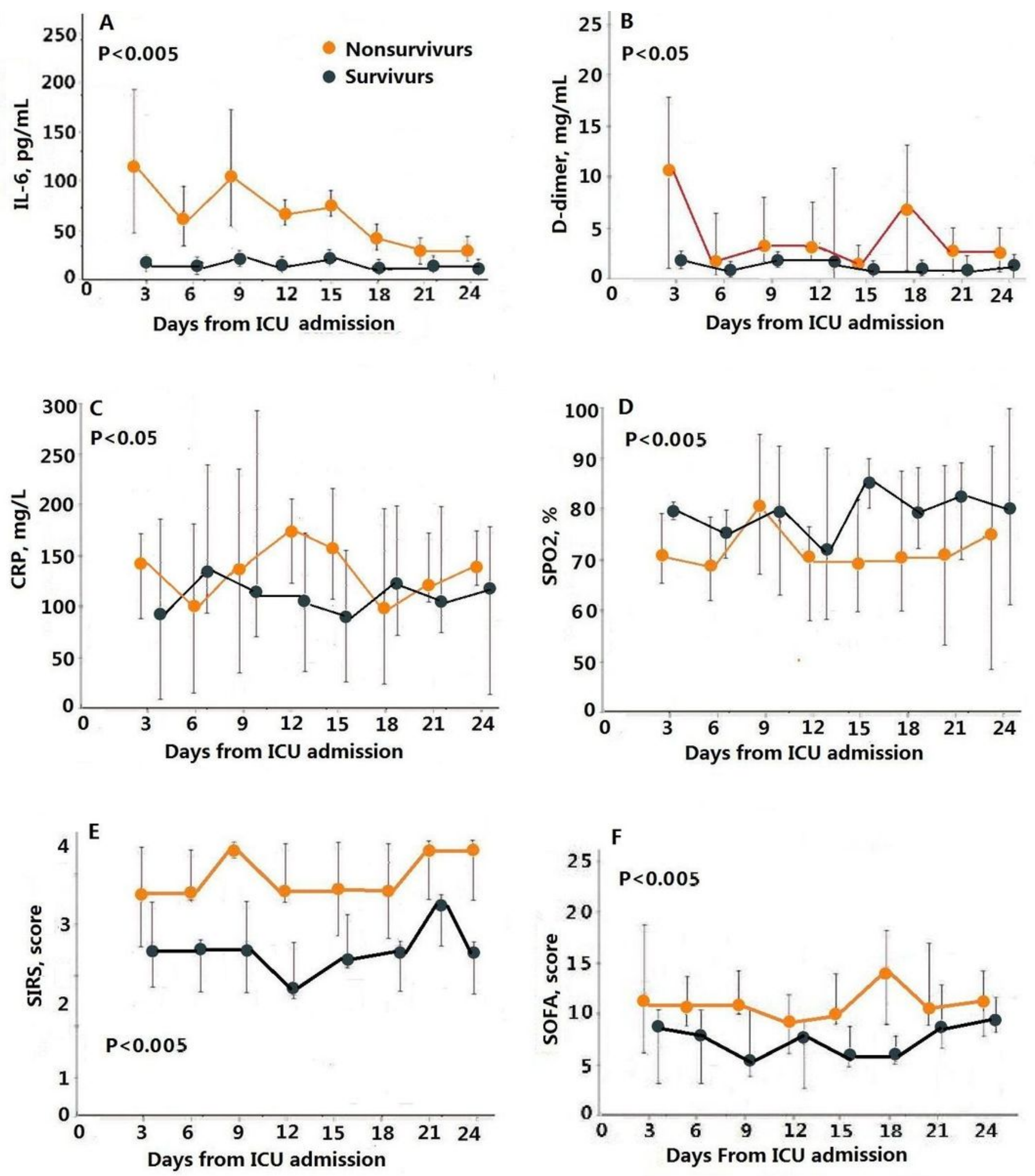

\section{Figure 3}

Temporal changes in laboratory parameters from nonoutbreak bacterial sepsis in the ICU. IL-6 (A), Ddimer (B), CRP (C), SPO2 (D), the SIRS score (E), and the SOFA score (F) were higher in nonsurvivors than in survivors (all $\mathrm{P}<0.05$ ). IL-6, Interleukin-6; CRP, C-reactive protein; Sp02, saturation of arterial oxygen; SIRS, systemic inflammatory response syndrome; SOFA, sequential [sepsis-related] organ function assessment. 


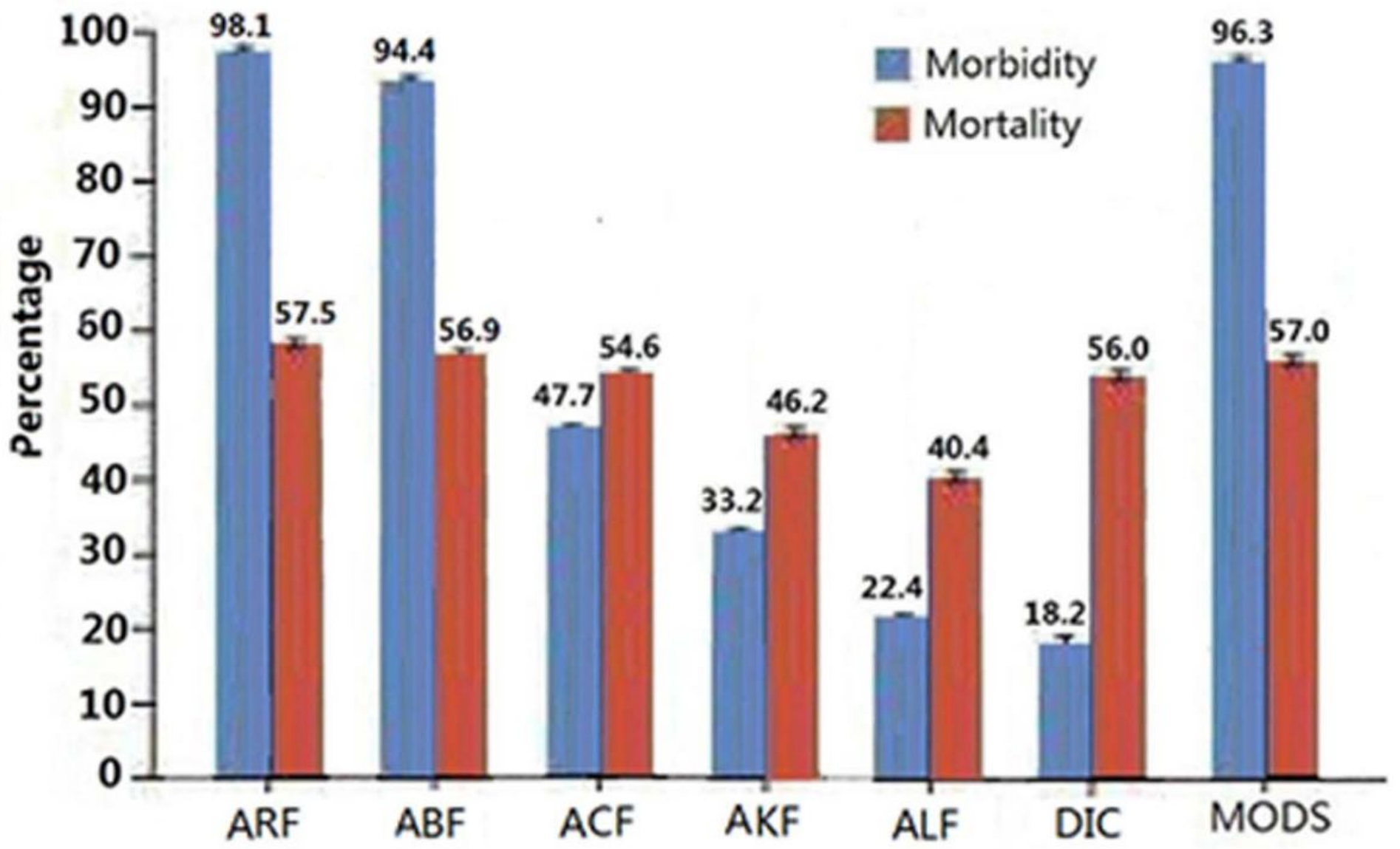

\title{
Risk of morbidity and mortality of 214 ICU patients with acute organ dysfunction, accrding to SOFA criteria
}

\begin{abstract}
ARF, acute hypoxemic respiratory fallure(AHRFyacute respiratorydistress syndrome(ARDS): ABF, acute brain Fallure; ACF, acute circulation fallure/shock: AKF, acute kidney fallure; ALF, acute liver fallure. DIC, disseminated intravascular coagulation; MODS, multiple organ dysfunction syndrome.
\end{abstract}

Figure 4

Risk of morbidity and mortality of 214 sepsis patients with acute organ dysfunctions in the ICU, according to the sepsis-sequential organ function assessment (SOFA). 